\title{
(6) OPEN ACCESS \\ Systemic pharmacokinetics following intravitreal injections of ranibizumab, bevacizumab or aflibercept in patients with neovascular AMD
}

\author{
Robert L Avery, ${ }^{1}$ Alessandro A Castellarin, ${ }^{1}$ Nathan C Steinle, ${ }^{1}$ Dilsher S Dhoot, ${ }^{1}$ \\ Dante Joseph Pieramici, ${ }^{1}$ Robert See, ${ }^{1}$ Stephen Couvillion, ${ }^{1}$ Ma'an A Nasir, ${ }^{1}$ \\ Melvin D Rabena, ${ }^{1}$ Kha Le, ${ }^{2}$ Mauricio Maia, ${ }^{2}$ Jennifer E Visich ${ }^{2}$
}

${ }^{1}$ California Retina Consultants,
Santa Barbara, California, USA
${ }^{2}$ Genentech Inc., South
San Francisco, California, USA

\section{Correspondence to}

Dr Robert L Avery, California Retina Consultants,

515 E. Micheltorena Street, Suite \#C, Santa Barbara,

CA 93103, USA;

bobave@gmail.com

Received 16 March 2014 Revised 23 May 2014 Accepted 21 June 2014 Published Online First 7 July 2014

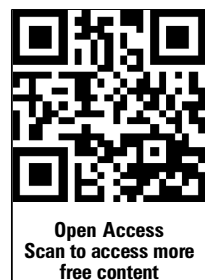

\section{ABSTRACT}

Background Data comparing systemic exposure and systemic vascular endothelial growth factor (VEGF) suppression of ranibizumab, bevacizumab and aflibercept following intravitreal injection are lacking.

Methods Fifty-six patients with wet age-related macular degeneration received intravitreal ranibizumab (0.5 mg), bevacizumab $(1.25 \mathrm{mg})$, or aflibercept (2.0 mg). Serum pharmacokinetics and plasma free VEGF were evaluated after the first and third injections.

Results Following the first dose, systemic exposure to aflibercept was 5-, 37-, and 9-fold higher than ranibizumab, whereas, bevacizumab was 9-, 310-, and 35-fold higher than ranibizumab, based on geometric mean ratio of peak and trough concentrations and area under the curve, respectively. The third dose showed accumulation of bevacizumab and aflibercept but not ranibizumab. Aflibercept substantially suppressed plasma free VEGF, with mean levels below lower limit of quantitation $(10 \mathrm{pg} / \mathrm{mL})$ as early as $3 \mathrm{~h}$ postdose until $\geq 7$ days postdose. Mean free (unbound) VEGF levels with ranibizumab were largely unchanged, with mean trough level of $14.4 \mathrm{pg} / \mathrm{mL}$ compared with baseline of $17 \mathrm{pg} / \mathrm{mL}$.

Conclusions There are notable differences in systemic pharmacokinetics and pharmacodynamics among antiVEGF treatments after intravitreal administration. All three agents rapidly moved into the bloodstream, but ranibizumab very quickly cleared, whereas bevacizumab and aflibercept demonstrated greater systemic exposure and produced a marked reduction in plasma free VEGF. Trial registration number NCT02118831.

\section{INTRODUCTION}

The discovery that anti-vascular endothelial growth factor (VEGF) agents injected intravitreally can reverse the anatomic and visual symptoms of neovascular (wet) age-related macular degeneration (AMD) revolutionised the treatment of wet AMD and other neovascular diseases of the retina. ${ }^{1}$ The safety and efficacy profiles of these drugs have been recognised in their adoption as first-line treatment for wet AMD. ${ }^{1}$

Three drugs - ranibizumab, bevacizumab and aflibercept-account for the vast majority of anti-VEGF injections, of which two, ranibizumab and aflibercept, were specifically developed for intravitreal administration and approved by the US Food and Drug Administration (FDA) for treatment of wet AMD. Ranibizumab is also approved in the
USA for treatment of macular oedema following retinal vein occlusion and diabetic macular oedema, and aflibercept is also approved in the USA for macular oedema following central retinal vein occlusion.

Ranibizumab, bevacizumab and aflibercept differ in their molecular weight, structure and pharmacokinetics. Bevacizumab, designed and developed to starve solid tumours of their blood supply by systemically inhibiting angiogenesis, is a $149 \mathrm{KDa}$ fulllength, bivalent monoclonal antibody against VEGF-A. ${ }^{2}$ It is salvaged from proteolytic catabolism and recycled via binding to $\mathrm{FcRn}$ in endothelial cells, resulting in a long systemic half-life of approximately 20 days following intravenous infusion. ${ }^{2}$ Ranibizumab is a $48 \mathrm{KDa}$ monovalent monoclonal antibody fragment, the antigen-binding Fab without the Fc domain. ${ }^{3}$ This structure was designed to prevent FcRn binding and, therefore, to dramatically shorten its systemic half-life to approximately $2 \mathrm{~h}$ after entering systemic circulation from the eye $\mathrm{e}^{4}$ and to facilitate distribution across all retinal layers to the choroidal vasculature. ${ }^{5}$ Aflibercept, by contrast, is a $115 \mathrm{KDa} \mathrm{Fc}$ fusion protein combining the binding domains of VEGF receptors 1 and 2 with an Fc antibody fragment, and was developed for intraocular injection and a systemic infusion. ${ }^{6}$ Because it has an intact Fc region, it is likely to be subject to FcRn recycling, which is supported by a serum half-life of approximately 5-6 days following intravenous administration. $^{7}$

Off-label use of bevacizumab is driven by cost-to-patient considerations and similar efficacy in several comparative clinical trials in wet AMD. ${ }^{8-13}$ Although visual outcome was non-inferior to ranibizumab in the Comparison of AMD Treatment Trials (CATT) trial, bevacizumab patients had a higher incidence of systemic serious adverse events (SAEs) at 1 and 2 years (OR 1.3), which was statistically significant at both time points. $^{12}{ }^{13}$ A meta-analysis of 2-year CATT and Inhibition of VEGF in Age-related Choroidal Neovascularisation (IVAN) studies showed a similar result (OR 1.32; 95\% CI, 1.08 to 1.59$).{ }^{8}$ While a comprehensive understanding of these findings is lacking as some of the SAEs are not typically associated with VEGF inhibition, the clinical experience with systemic VEGF inhibition in oncology has established that systemic VEGF inhibition is associated with several 'class' adverse effects, including hypertension, proteinuria, arterial thromboembolic events, 
cardiomyopathy, haemorrhage, wound healing complications, gastrointestinal perforation, and reversible posterior leukoencephalopathy syndrome. ${ }^{14}$ Even though only small amounts of the anti-VEGF drugs are released from the eye into the systemic circulation compared with doses used in oncology, these agents are very potent, with $\mathrm{IC}_{50}$ values in the subnanomolar range, and systemic levels that appear sufficient to suppress circulating VEGF. ${ }^{15-18}$ In the IVAN study, the decrease in serum free VEGF from baseline at 12 months and 24 months was significantly greater with bevacizumab compared with ranibizumab. ${ }^{9} 19$

The vitreous half-life of ranibizumab in patients with neovascular AMD estimated from the serum data following intravitreal injection in patients with neovascular AMD was 9 days in a population pharmacokinetic (PK) model. ${ }^{4}$ On reaching the systemic circulation, ranibizumab is cleared with an elimination half-life of approximately $2 \mathrm{~h}^{4}{ }^{4}$ By directly measuring aqueous half-lives, the vitreous half-lives of ranibizumab and bevacizumab have been estimated to be 7.2 and 9.8 days. $^{20} 21$ Comparable data for bevacizumab and aflibercept are lacking, as are studies comparing the systemic levels of ranibizumab, bevacizumab and aflibercept, and their relative effects on circulating VEGF. In the present prospective study, we evaluated serum drug levels and plasma free VEGF levels in patients with neovascular AMD following intravitreal injections of ranibizumab, bevacizumab, or aflibercept.

\section{METHODS}

\section{Study design}

This prospective study enrolled patients with neovascular AMD who were naive to anti-VEGF therapy or had not received intravitreal anti-VEGF therapy for the previous 4 months. Patients were excluded if they were unwilling to receive three monthly injections of anti-VEGF as initial therapy, had received systemic anti-VEGF for cancer treatment in the past year, were on dialysis, or had undergone vitrectomy. Consecutive eligible patients seen in a single private practice, California Retinal Consultants (CRC), were sequentially offered enrolment into the study. Fifteen AMD patients were allocated to the bevacizumab group, 20 to the ranibizumab group, and 21 to the aflibercept group $(\mathrm{N}=56)$. Each study participant received 3 monthly intravitreal injections of ranibizumab $0.5 \mathrm{mg}$, bevacizumab $1.25 \mathrm{mg}$, or aflibercept $2.0 \mathrm{mg}$ (all sourced commercially, with Avastin provided by a single compounding pharmacy) as part of the normal course of their retinal care. All patients provided written informed consent for study participation. This study received Institutional Review Board approval and was conducted in accordance with current US FDA Good Clinical Practices (GCP) and local ethical and legal requirements. Blood samples were collected at screening, $3 \mathrm{~h}$ postinjection, and days 1, 3, 7 and 28, following doses 1 and 3 for PK and systemic VEGF analysis.

The primary study objective was to assess systemic drug PK and free (unbound) VEGF for anti-VEGF-treated patients after the first and third doses of therapy. A secondary objective was to evaluate differences in PK and free VEGF between molecules. Outcome measures included serum PK and plasma free VEGF levels for ranibizumab, bevacizumab and aflibercept, following dose 1 and dose 3 at baseline, $3 \mathrm{~h}$ postinjection, and days 1, 3, 7 and 28 .

\section{Sample collection and analysis}

Plasma samples for free VEGF analysis were collected using CTAD (citrate, theophylline, adenosine, dipyridamole) tubes, which contain a combination of anticoagulant agents shown to prevent platelet activation, minimising the release of platelet- derived growth factors and cytokines, including VEGF. ${ }^{22}$ Serum samples were obtained for drug PK analysis. Blood $(6 \mathrm{~mL})$ was drawn on-site at CRC, centrifuged, and frozen at $-70^{\circ} \mathrm{C}$, on-site within $2 \mathrm{~h}$ of collection, and kept frozen until shipping. Blood samples for plasma free VEGF were collected and shipped to Covance (Princeton, New Jersey, USA), where samples were banked and shipped in bulk to Pharmaceutical Product Development, LLC (PPD; Wilmington, North Carolina, USA) for analysis. Sample analysis was performed by PPD staff blinded to study information other than treatment allocation. PK analyses were performed by Genentech.

Serum ranibizumab, bevacizumab and aflibercept concentrations were determined using ELISA in which samples were preincubated with molar excess of recombinant-human VEGF-A, followed by capture and detection using specific monoclonal antibody reagents that bind quantitatively to VEGF-drug complexes. The ranibizumab assay was validated, while the bevacizumab and aflibercept assays were fully qualified. The three assays were shown to accurately and reproducibly quantify VEGF-bound and unbound species of each anti-VEGF agent (ie, total drug present in the samples). The lower limit of quantitation (LLOQ) of the ranibizumab, bevacizumab and aflibercept assays were $15.0,313$, and $1000 \mathrm{pg} / \mathrm{mL}$, respectively. Plasma concentrations of free VEGF were determined using Quantikine ELISA kit (R\&D Systems, Minneapolis, Minnesota, USA). The kit was used as described by the manufacturer, except that an additional point was added to the calibration curve at $7.8 \mathrm{pg} / \mathrm{mL}$ (a twofold dilution of the last standard in the kit) in order to enable the assay to accurately quantify VEGF concentrations at approximately $10 \mathrm{pg} / \mathrm{mL}$; phosphate buffered saline containing $0.05 \%$ Tween-20 was used to wash plates between incubation steps, replacing 'Wash Buffer 1' due to the large number of samples; and 3,3',5,5'-tetramethylbenzidine (TMB) substrate incubation time was evaluated between 25 and 30 min compared with the kit instructions of 25 min. With these minor modifications, the resulting assay procedure passed qualification per manufacturer specifications and performed well during sample testing.

Plasma VEGF was measured rather than serum VEGF, in order to exclude measurement of the VEGF sequestered in platelets, and to better approximate the VEGF concentrations to which the endothelial cells are exposed.

\section{Statistical and PK analyses}

No formal sample size calculation was performed, and the sample size was chosen to ensure study completion within 1 year. Plasma/serum were collected at predetermined time points at baseline, $3 \mathrm{~h}$ postinjection, and days 1, 3, 7 and 28 following dose 1 and dose 3 . Concentration data were analysed using non-compartmental $\mathrm{PK}$ to determine area under the curve (AUC), peak concentration $\left(\mathrm{C}_{\max }\right)$, and trough concentration $\left(\mathrm{C}_{\min }\right)$. Descriptive statistics were used to summarise the results with no formal hypothesis testing. Serum anti-VEGF drug concentrations and plasma VEGF levels that were below the LLOQ were imputed to one-half the LLOQ value.

\section{RESULTS}

\section{Patients}

Fifty-six patients (29 male, 27 female) with neovascular AMD were enrolled. The mean age of the patients in the bevacizumab and aflibercept arms was 75 years, and 77 years in the ranibizumab arm. 


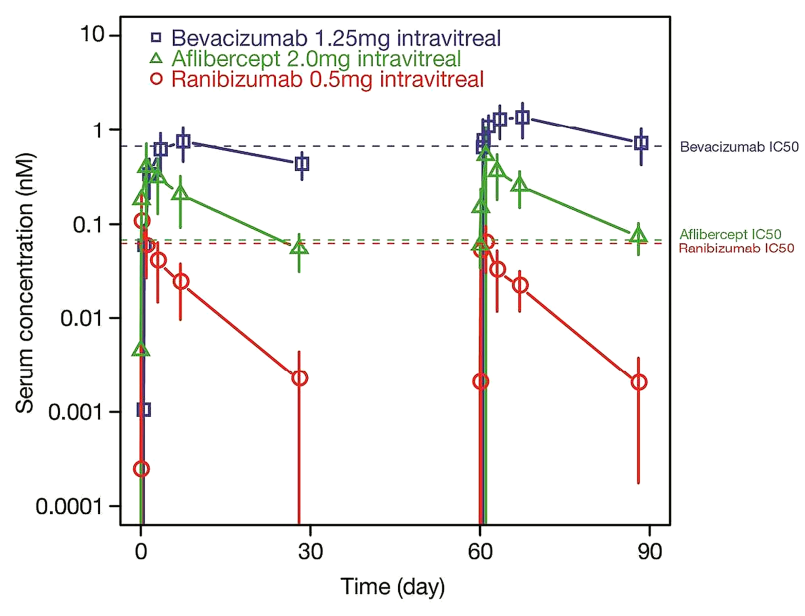

Figure 1 Serum concentration-time curves for ranibizumab, bevacizumab, or aflibercept following intravitreal injection in patients with age-related macular degeneration.

\section{Systemic exposure of ranibizumab, bevacizumab and aflibercept}

Systemic exposure to intravitreal administration of ranibizumab, aflibercept and bevacizumab was assessed by $\mathrm{C}_{\max }$, AUC over 28 days $\left(\mathrm{AUC}_{0-28}, \mathrm{AUC}_{60-88}\right.$ ), and $\mathrm{C}_{\min }$ at 1 month after dosing. Mean (SD) serum concentration-time profiles after intravitreal injection of $0.5 \mathrm{mg}$ ranibizumab, $2.0 \mathrm{mg}$ aflibercept and $1.25 \mathrm{mg}$ bevacizumab are presented in figure 1 . Systemic exposure data are summarised in table 1 .

Following the first intravitreal injection on day $0, \mathrm{C}_{\max }$ was reached at median time to $\mathrm{C}_{\max }\left(\mathrm{t}_{\max }\right)$ of $0.9,1.1$ and 7.0 days for ranibizumab, aflibercept and bevacizumab, respectively. Among the three drugs, systemic exposure to bevacizumab was highest, whereas that of ranibizumab was lowest. Based on the geometric mean ratio of $\mathrm{C}_{\max }, \mathrm{C}_{\min }$, and AUC, respectively, following first dose, systemic exposure to aflibercept was 5 -fold, 37-fold and 9-fold higher than ranibizumab, whereas that of bevacizumab was 9-fold, 310-fold and 35-fold higher than ranibizumab. Following the third dose, the differences between aflibercept versus ranibizumab and bevacizumab versus ranibizumab increased further; based on the geometric mean ratio of $\mathrm{C}_{\max }, \mathrm{C}_{\min }$ and AUC, respectively, systemic exposure to aflibercept was 7-fold, 53 -fold and 14-fold higher than ranibizumab, whereas, that of bevacizumab was 23 -fold, 500 -fold and 72 -fold higher than ranibizumab.

No accumulation was observed between the first and third doses of ranibizumab, whereas systemic accumulation was observed for aflibercept and bevacizumab. Accumulation ratio between the third and first doses were 1.37 (95\% CI 1.03 to 1.83), 1.19 (90\% CI 0.77 to 1.84 ), and 1.27 (95\% CI 1.01 to 1.59) for aflibercept and 1.56 (95\% CI 1.15 to 2.12), 1.95 (95\% CI 1.43 to 2.68 ), and 1.84 (95\% CI 1.37 to 2.48 ) for bevacizumab, based on the dose 3 /dose 1 geometric mean ratio of $\mathrm{C}_{\min }, \mathrm{C}_{\max }$, and $\mathrm{AUC}_{28}$, respectively.

When comparing the in vitro $\mathrm{IC}_{50}$ values for VEGF based on bovine retinal microvascular endothelial cell proliferation assay, mean serum bevacizumab concentrations were higher than its $\mathrm{IC}_{50}\left(0.668 \mathrm{nM}^{23}\right)$ at day 3 and day 7 after the first injection, and at all observed time points after the third injection through day 88. Mean aflibercept serum concentrations exceeded its $\mathrm{IC}_{50}$ value $\left(0.068 \mathrm{nM}^{23}\right)$ at $3 \mathrm{~h}$ postdose, and remained above $\mathrm{IC}_{50} \geq 7$ days postdose. By contrast, mean ranibizumab serum concentrations exceeded its $\mathrm{IC}_{50}\left(0.060 \mathrm{nM}^{23}\right)$ transiently at $\mathrm{C}_{\max }$ and returned below the $\mathrm{IC}_{50}$ by 1-2 days postinjection.

\section{Free systemic VEGF levels}

The mean baseline free VEGF levels were $22.5 \mathrm{pg} / \mathrm{mL}$ (95\% CI 17.3 to $27.6 \mathrm{pg} / \mathrm{mL}$ ), $19.2 \mathrm{pg} / \mathrm{mL}$ (95\% CI 15.3 to $23.1 \mathrm{pg} / \mathrm{mL}$ ) and $17.0 \mathrm{pg} / \mathrm{mL}$ (95\% CI 11.6 to $22.5 \mathrm{pg} / \mathrm{mL}$ ) for the bevacizumab, aflibercept and ranibizumab arms, respectively. There is considerable variability at the individual patient level as demonstrated by the box plots in figure 3. In general, these baseline VEGF levels are somewhat lower than those reported in similar studies. This is likely due to the fact that the current study used CTAD tubes and other best practices for plasma collection, thereby minimising platelet activation and/or rupture, which would be expected to result in higher levels of measured VEGF. The appearance of VEGF inhibitors in the systemic circulation was paralleled by differential reduction in the levels of free (unbound) plasma VEGF. Intravitreal bevacizumab appeared to

Table 1 Mean (SD) systemic exposures of bevacizumab, ranibizumab and aflibercept

\begin{tabular}{|c|c|c|c|c|c|}
\hline & \multirow[b]{2}{*}{ Bevacizumab } & \multirow[b]{2}{*}{ Ranibizumab } & \multirow[b]{2}{*}{ Aflibercept } & \multicolumn{2}{|c|}{ Geometric mean ratio } \\
\hline & & & & $\begin{array}{l}\text { Bevacizumab/ } \\
\text { ranibizumab } \\
(95 \% \mathrm{Cl})\end{array}$ & $\begin{array}{l}\text { Aflibercept/ } \\
\text { ranibizumab } \\
(95 \% \mathrm{Cl})\end{array}$ \\
\hline \multicolumn{6}{|l|}{ First dose } \\
\hline$C_{\max }, \mathrm{nM}$ & $\begin{array}{l}0.76(0.31) \\
n=15\end{array}$ & $\begin{array}{l}0.11(0.13) \\
n=20\end{array}$ & $\begin{array}{l}0.45(0.29) \\
n=21\end{array}$ & 8.80 (5.59 to 13.8 ) & 4.65 (3.07 to 7.05$)$ \\
\hline $\mathrm{C}_{\min ,} \mathrm{nM}$ & $\begin{array}{l}0.44(0.14) \\
\mathrm{n}=14\end{array}$ & $\begin{array}{l}0.002(0.002) \\
n=19\end{array}$ & $\begin{array}{l}0.05(0.02) \\
\mathrm{n}=20\end{array}$ & 310 (188 to 511$)$ & 37.3 (23.7 to 58.7 ) \\
\hline $\mathrm{AUC}_{0-28}, \mathrm{nM}^{*} \mathrm{~h}$ & $\begin{array}{l}15.73(5.76) \\
n=14\end{array}$ & $\begin{array}{l}0.46(0.24) \\
n=19\end{array}$ & $\begin{array}{l}4.32(1.77) \\
n=20\end{array}$ & 34.9 (26.4 to 46.1$)$ & 9.49 (7.4 to 12.2$)$ \\
\hline \multicolumn{6}{|l|}{ Third dose } \\
\hline $\mathrm{C}_{\max }, \mathrm{nM}$ & $\begin{array}{l}1.47(0.55) \\
n=15\end{array}$ & $\begin{array}{l}0.07(0.05) \\
\mathrm{n}=18\end{array}$ & $\begin{array}{l}0.58(0.52) \\
n=21\end{array}$ & 22.7 (14.8 to 34.8 ) & 7.28 (4.91 to 10.8 ) \\
\hline $\mathrm{C}_{\min ,} \mathrm{nM}$ & $\begin{array}{l}0.70(0.29) \\
n=14\end{array}$ & $\begin{array}{l}0.002(0.002) \\
n=18\end{array}$ & $\begin{array}{l}0.07(0.03) \\
n=21\end{array}$ & 500 (304 to 822) & 52.9 (33.8 to 82.8$)$ \\
\hline $\mathrm{AUC}_{60-88,}, \mathrm{nM}^{*} \mathrm{~h}$ & $\begin{array}{l}29.12(10.35) \\
n=14\end{array}$ & $\begin{array}{l}0.41(0.17) \\
n=18\end{array}$ & $\begin{array}{l}5.38 \\
n=21\end{array}$ & 72.4 (55.4 to 94.8 ) & 13.5 (10.6 to 17.3$)$ \\
\hline
\end{tabular}

AUC, area under curve; $C_{\max }$ maximum serum concentration; $C_{\min }$, minimum serum concentration. 


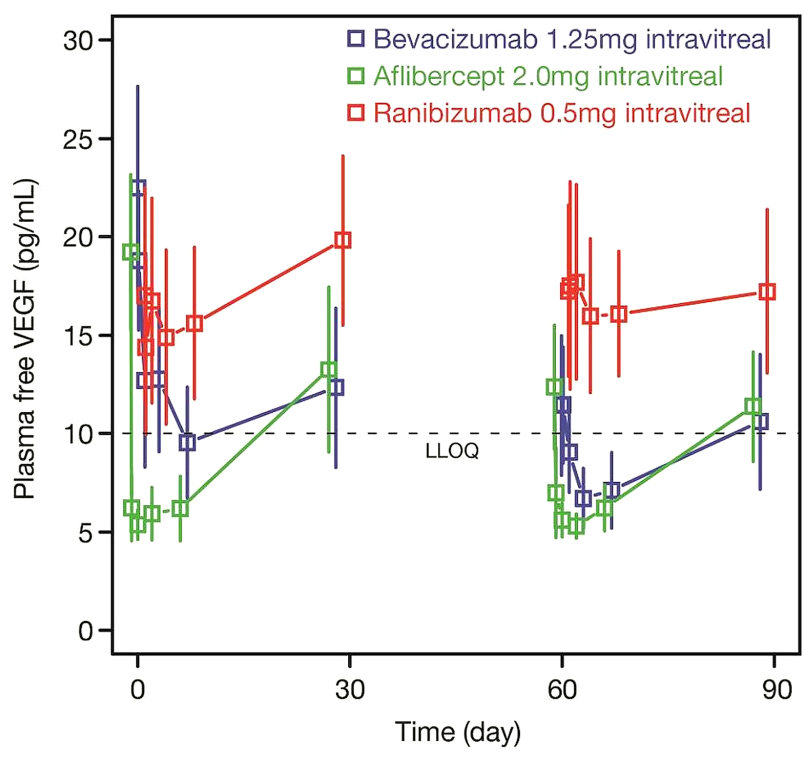

Figure 2 Mean $(95 \% \mathrm{Cl})$ plasma free VEGF concentration following intravitreal injection of ranibizumab, bevacizumab, or aflibercept in patients with age-related macular degeneration. VEGF, vascular endothelial growth factor.

produce significant suppression of free plasma VEGF to a mean $\mathrm{C}_{\text {min }}$ of $\sim 10 \mathrm{pg} / \mathrm{mL}$ following the first dose, and to below the LLOQ between 1 and 7 days following the third dose (figure 2). This increased suppression of free VEGF levels was associated with higher measured serum concentrations of bevacizumab following the third dose.

Intravitreal aflibercept showed the greatest suppression of free plasma VEGF, with the vast majority of samples below the LLOQ after the first and third doses (figure 2). Mean free VEGF levels dropped below LLOQ as early as $3 \mathrm{~h}$ postdose and remained below LLOQ until $\geq 7$ days postdose.

Mean free (unbound) VEGF levels following ranibizumab injections were largely unchanged, with only a minimal drop compared with baseline level. Mean trough levels of free VEGF were $\sim 14.4 \mathrm{pg} / \mathrm{mL}$, compared with a baseline of $17 \mathrm{pg} / \mathrm{mL}$.

Individual patient values (figure 3 ) for aflibercept showed dramatic suppression of free VEGF through day 7, with most samples showing suppression to the LLOQ. Patient values for bevacizumab showed large suppression of free VEGF as well, somewhat less than aflibercept, which was more pronounced after the third dose. Patient values for ranibizumab showed no obvious trend after the first or third dose.

\section{DISCUSSION}

The introduction of intravitreal anti-VEGF agents has provided improved outcomes producing a paradigm shift in the treatment of many retinal diseases. Despite the similar visual outcome in many of the head-to-head comparative trials of bevacizumab, ranibizumab and aflibercept in wet $\mathrm{AMD}$, there are significant differences in the molecular structure of these agents, resulting in very different PK profiles, such as systemic exposure after intravitreal injection.

In the bloodstream, Fc-containing molecules are recycled by binding endothelial cell FcRn receptors to protect them from the default degradative pathway within endosomes. ${ }^{24}$ This recycling decreases the rate of systemic clearance of Fc-containing molecules, such as bevacizumab and aflibercept. Ranibizumab, by contrast, was designed without the Fc domain to allow for rapid systemic clearance. The serum PK profiles of ranibizumab, bevacizumab and aflibercept observed in the present study are consistent with these differences. Ranibizumab appeared transiently in systemic circulation and was rapidly cleared, with the lowest total systemic exposure as measured by AUC. Bevacizumab, by contrast, was cleared relatively slowly from systemic circulation and appeared to accumulate with repeated dosing. Aflibercept accumulated in systemic circulation less than bevacizumab, but had a systemic exposure (AUC) an order of magnitude greater than ranibizumab.

Ranibizumab had little effect on plasma free VEGF concentrations, whereas bevacizumab significantly reduced free VEGF in plasma, and did so to a greater extent after the third injection, presumably as a result of the observed systemic accumulation of bevacizumab. These findings are consistent with those reported by Carneiro et $a l^{15}$ and Zehetner et $a l^{18}$ in which bevacizumab, but not ranibizumab, was associated with significantly reduced plasma free VEGF concentrations 28 days postintravitreal injection in patients with wet AMD. Measurement of plasma free VEGF can be challenging, as platelet rupture and/or activation can lead to release of intracellular VEGF, resulting in increased VEGF levels. The use of CTAD collection tubes ensured that this artefact was minimised. Consequently, our baseline VEGF levels are less than in many similar studies. Another concern with measurements of free VEGF in the presence of anti-VEGF agents (with different dissociation rates) is that VEGF bound to these agents may dissociate differentially during the assay, and thus, affect the measurements. However, the net effect of such process would be an overestimation of free VEGF levels, and furthermore, published data on dissociation rates would not explain the marked difference in reduction of free VEGF levels we observed between ranibizumab and aflibercept patients. ${ }^{25}$ Furthermore, the IVAN study, which measured serum free VEGF, also found a significant reduction in free VEGF levels among patients treated with bevacizumab, more so than in those treated with ranibizumab. ${ }^{9}{ }^{19}$ Despite its serum concentrations intermediate between ranibizumab and bevacizumab, aflibercept showed the greatest reduction in plasma free VEGF, most likely because of the higher affinity of aflibercept for VEGF compared with bevacizumab. ${ }^{25}$ In this study, which is limited by its small sample size and lack of formal statistical analysis, it is nevertheless noteworthy that the marked reductions in plasma VEGF correlate with the elevation of mean systemic drug levels above their $\mathrm{IC}_{50}$ for VEGF. The reduction in free VEGF parallels the changes in the serum concentrations of the different inhibitor molecules, and this consistency makes it unlikely that the changes and patterns seen are artefacts for events such as random release of VEGF from platelets.

The movement of aflibercept and bevacizumab into the bloodstream, and the associated reduction in plasma free VEGF was very rapid, seen at $3 \mathrm{~h}$ post-treatment. Although this may seem unexpected given the large size of these molecules, Kim et $a l^{26}$ described a mechanism by which the neonatal Fc receptor facilitates the transport of Fc-containing molecules from the vitreous into the retinal bloodstream.

The registration and comparative trials of ranibizumab and aflibercept have demonstrated a good safety profile without any statistically significant systemic increased risk of arterial thrombolic embolism. However, there have been hints that a safety concern could potentially arise with larger numbers of patients studied or longer follow-up. A meta-analysis of five early AMD trials demonstrated an increased risk of cerebrovascular event if ranibizumab patients were stratified by baseline stroke risk. ${ }^{27}$ Furthermore, in the second year of the RIDE (NCT00473382) 

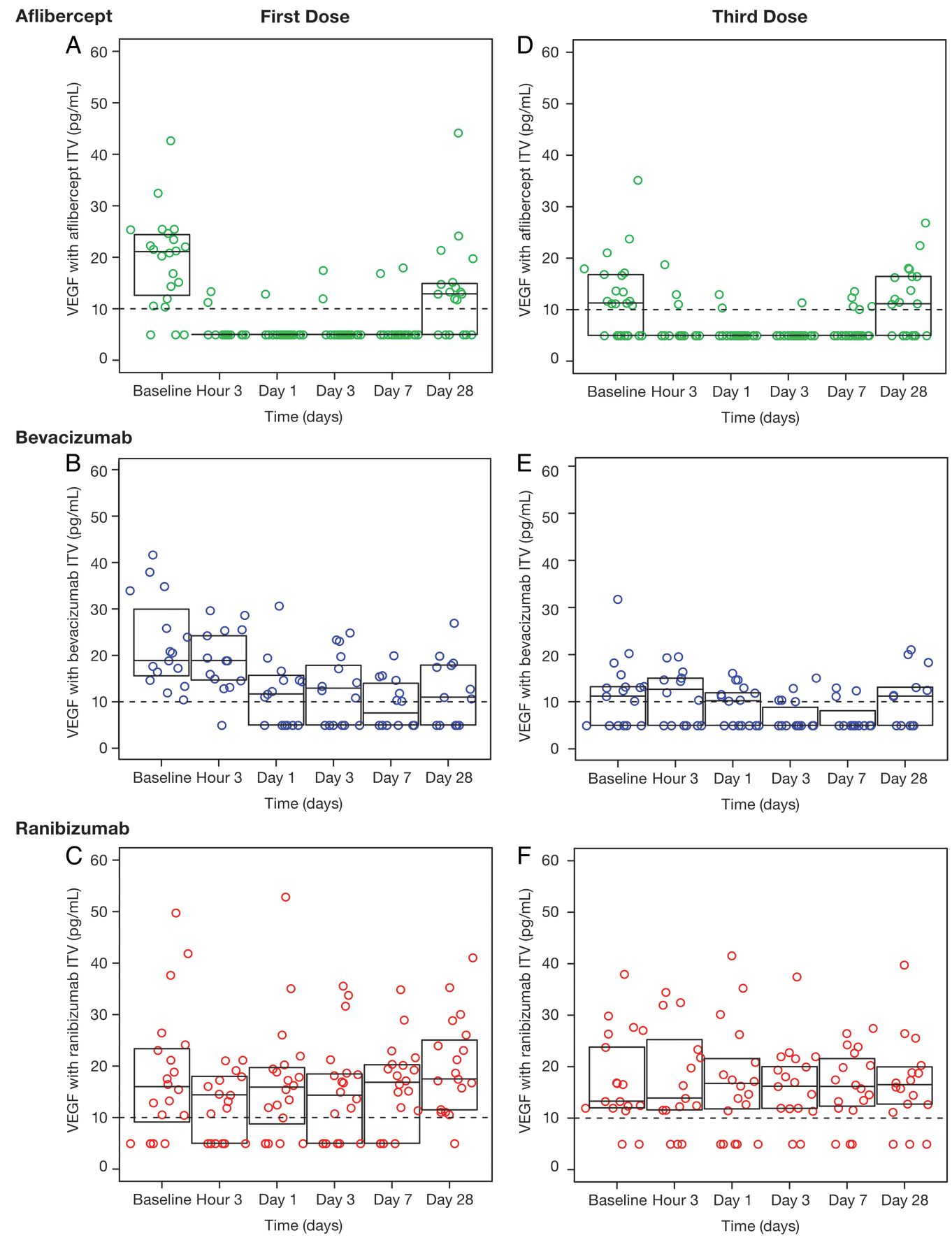

Figure 3 Individual observed plasma free VEGF concentrations following intravitreal injection of ranibizumab, bevacizumab, or aflibercept. ITV, intravitreal; VEGF, vascular endothelial growth factor.

and RISE (NCT00473330) trials of ranibizumab for diabetic macular oedema, an imbalance was noted with deaths and CVAs in the higher dose arms which prompted the approval of the lower, $0.3 \mathrm{mg}$, dose for diabetic macular oedema in the USA. ${ }^{28}$ Interestingly, in the second year of the VISTA trial of aflibercept for diabetic macular oedema, a similar increased incidence of death was reported in the monthly arm with the higher cumulative dose. ${ }^{28}$ As previously mentioned, the CATT trial identified an increased risk of systemic SAEs, albeit not always thought to be VEGF mediated, with bevacizumab over ranibizumab, and further meta-analyses have shown a similar increased risk. ${ }^{8} 12$ Although statistically significant in the large CATT trial, this difference has not been consistent in the smaller European comparative trials. In addition, the European Medicines Agency European Public Assessment Report of aflibercept showed a potential signal for increased cerebrovascular events, many of which were transient ischaemic attacks, in elderly patients with aflibercept compared with ranibizumab, but a slightly lower rate of cardiovascular events. ${ }^{29}$ As useful as these head-to-head studies have been, none has the duration of follow-up or the statistical power to detect differences in infrequent systemic adverse events against the noise inherent in studies conducted in a predominantly elderly population. Other observations lending evidence for possible systemic effects of intravitreal anti-VEGF agents are the numerous fellow eye effects that have been reported, especially with bevacizumab. ${ }^{30} 31$ 
VEGF inhibitors have a well-known systemic safety and tolerability profile due to their use in oncology that outlines a plausible biological explanation for potential systemic SAEs observed in some recent ophthalmology trials, potentially related to reduced systemic VEGF levels. ${ }^{32}$ The results we report here show differences among the three main intravitreal VEGF inhibitors in their systemic PK and pharmacodynamics that may provide biological plausibility for potential differences in systemic safety risk.

Acknowledgements The authors thank Ai Ping Lee and Xin Wang for assistance with data reconciliation and analysis. Support for third-party medical writing and editorial assistance was provided by Genentech Inc.

Contributors RLA conceived and designed the study. AAC, NCS, DSD, DJP, RS, SC, and MAN contributed important intellectual content to the study design and manuscript, and treated study patients. MDR coordinated sample collection, contributed to data collection, and contributed to the manuscript. KL, MM, and JEV designed and validated the analysis methods, performed sample quantitation, analysed data, and contributed to manuscript writing. Ai Ping Lee and Xin Wang (acknowledged) contributed significantly to data reconciliation and analysis. William E Watkins of Envision Scientific Solutions (third party medical writer) drafted the manuscript under direction from Dr Avery.

Funding This investigator-sponsored trial was partially funded through a grant from Genentech, Inc (ML28032). Presented in part at the American Society of Retina Specialists, Toronto, Canada; American Academy of Ophthalmology, New Orleans; Angiogenesis, Exudation, and Degeneration, Miami Florida; and Macula Society, Key Largo, Florida.

Competing interests RLA reports grants and non-financial support from Genentech, during the conduct of the study; grants, personal fees and non-financial support from Genentech, personal fees from Alcon, personal fees from Alimera, grants and personal fees from Allergan, grants, personal fees and non-financial support from Regeneron, personal fees and non-financial support from Replenish, grants and personal fees from Ophthotech, personal fees and non-financial support from Novartis, personal fees from Johnson and Johnson, grants and personal fees from QLT, personal fees from Bausch and Lomb, outside the submitted work; In addition, RLA has a patent Intravitreal Drug Delivery licensed to Replenish. DJP reports grants and non-financial support from Genentech, during the conduct of the study; grants and personal fees from Genentech, grants and personal fees from Allergan, personal fees from Santen, personal fees from Alimera, outside the submitted work. NCS reports grants and non-financial support from Genentech, during the conduct of the study; personal fees from Regeneron, outside the submitted work. AAC and SC report grants and non-financial support from Genentech, during the conduct of the study; grants and personal fees from Genentech, outside the submitted work. DSD, RS, MAN, and MDR report grants and non-financial support from Genentech, during the conduct of the study. KL, MM, and JEV are employees of Genentech.

Ethics approval Western IRB based in Olympia, WA.

Provenance and peer review Not commissioned; externally peer reviewed.

Open Access This is an Open Access article distributed in accordance with the Creative Commons Attribution Non Commercial (CC BY-NC 3.0) license, which permits others to distribute, remix, adapt, build upon this work non-commercially, and license their derivative works on different terms, provided the original work is properly cited and the use is non-commercial. See: http://creativecommons.org/ licenses/by-nc/3.0/

\section{REFERENCES}

1 Rothen $\mathrm{M}$, Jablon $\mathrm{E}$, Monares $\mathrm{G}$, et al. Anti-macular degeneration agents. Ophthalmol Clin North Am 2005;18:561-7.

2 Avastin (bevacizumab) solution for intravenous infusion prescribing information. http://www.gene.com/download/pdf/avastin_prescribing.pdf (accessed 11 Mar 2014).

3 Lucentis (ranibizumab injection) intravitreal injection prescribing information. http:/l www.gene.com/download/pdf/lucentis_prescribing.pdf (accessed 11 Mar 2014).

$4 \mathrm{Xu} \mathrm{L,} \mathrm{Lu} \mathrm{T,} \mathrm{Tuomi} \mathrm{L,} \mathrm{et} \mathrm{al.} \mathrm{Pharmacokinetics} \mathrm{of} \mathrm{ranibizumab} \mathrm{in} \mathrm{patients} \mathrm{with}$ neovascular age-related macular degeneration: a population approach. Invest Ophthalmol Vis Sci 2013:54:1616-24

5 Ferrara N, Damico L, Shams N, et al. Development of ranibizumab, an anti-vascular endothelial growth factor antigen binding fragment, as therapy for neovascular age-related macular degeneration. Retina 2006;26:859-70.
6 Stewart MW. Aflibercept (VEGF-TRAP): the next anti-VEGF drug. Inflamm Allergy Drug Targets 2011;10:497-508.

7 Holash J, Davis S, Papadopoulos N, et al. VEGF-Trap: a VEGF blocker with potent antitumor effects. Proc Natl Acad Sci USA 2002:99:11393-8.

8 Chakravarthy U, Harding SP, Rogers CA, et al. Alternative treatments to inhibit VEGF in age-related choroidal neovascularisation: 2-year findings of the IVAN randomised controlled trial. Lancet 2013;382:1258-67.

9 Chakravarthy U, Harding SP, Rogers CA, et al. Ranibizumab versus bevacizumab to treat neovascular age-related macular degeneration: one-year findings from the IVAN randomized trial. Ophthalmology 2012;119:1399-411.

10 Kodjikian L, Souied EH, Mimoun G, et al. Ranibizumab versus bevacizumab for neovascular age-related macular degeneration: results from the GEFAL noninferiority randomized trial. Ophthalmology 2013;120:2300-9.

11 Krebs I, Schmetterer L, Boltz A, et al. A randomised double-masked trial comparing the visual outcome after treatment with ranibizumab or bevacizumab in patients with neovascular age-related macular degeneration. Br I Ophthalmol 2013:97:266-71.

12 Martin DF, Maguire MG, Fine SL, et al.; Comparison of Age-related Macular Degeneration Treatments Trials (CATT) Research Group. Ranibizumab and bevacizumab for treatment of neovascular age-related macular degeneration: two-year results. Ophthalmology 2012;119:1388-98.

13 Martin DF, Maguire MG, Ying GS, et al. Ranibizumab and bevacizumab for neovascular age-related macular degeneration. N Engl J Med 2011;364:1897-908.

14 Chen HX, Cleck JN. Adverse effects of anticancer agents that target the VEGF pathway. Nat Rev Clin Oncol 2009;6:465-77.

15 Carneiro AM, Costa R, Falcao MS, et al. Vascular endothelial growth factor plasma levels before and after treatment of neovascular age-related macular degeneration with bevacizumab or ranibizumab. Acta Ophthalmol 2012;90:e25-30.

$16 \mathrm{Gu} \mathrm{X,} \mathrm{Yu} \mathrm{X,} \mathrm{Dai} \mathrm{H.} \mathrm{Intravitreal} \mathrm{Injection} \mathrm{of} \mathrm{ranibizumab} \mathrm{for} \mathrm{treatment} \mathrm{of} \mathrm{age-related}$ macular degeneration: effects on serum VEGF concentration. Curr Eye Res 2014:39:518-21.

17 Matsuyama K, Ogata N, Matsuoka M, et al. Plasma levels of vascular endothelial growth factor and pigment epithelium-derived factor before and after intravitreal injection of bevacizumab. Br J Ophthalmol 2010;94:1215-18.

18 Zehetner C, Kirchmair R, Huber S, et al. Plasma levels of vascular endothelial growth factor before and after intravitreal injection of bevacizumab, ranibizumab and pegaptanib in patients with age-related macular degeneration, and in patients with diabetic macular oedema. Br J Ophthalmol 2013;97:454-9.

19 Harding SP. IVAN Outcomes [abstract]. Invest Ophthalmol Vis Sci 2013;54. ARVO E-Abstract.

20 Krohne TU, Liu Z, Holz FG, et al. Intraocular pharmacokinetics of ranibizumab following a single intravitreal injection in humans. Am J Ophthalmol 2012;154:682-6 e2.

21 Krohne TU, Eter N, Holz FG, et al. Intraocular pharmacokinetics of bevacizumab after a single intravitreal injection in humans. Am J Ophthalmol 2008;146:508-12.

22 Aguilar-Mahecha A, Kuzyk MA, Domanski D, et al. The effect of pre-analytical variability on the measurement of MRM-MS-based mid- to high-abundance plasma protein biomarkers and a panel of cytokines. PLOS ONE 2012;7:e38290.

23 Yu L, Liang XH, Ferrara N. Comparing protein VEGF inhibitors: in vitro biological studies. Biochem Biophys Res Commun 2011:408:276-81.

24 Ternant D, Paintaud G. Pharmacokinetics and concentration-effect relationships of therapeutic monoclonal antibodies and fusion proteins. Expert Opin Biol Ther 2005;5(Suppl 1):S37-47.

25 Papadopoulos N, Martin J, Ruan Q, et al. Binding and neutralization of vascular endothelial growth factor (VEGF) and related ligands by VEGF Trap, ranibizumab and bevacizumab. Angiogenesis 2012;15:171-85.

26 Kim H, Robinson SB, Csaky KG. FcRn receptor-mediated pharmacokinetics of therapeutic IgG in the eye. Mol Vis 2009;15:2803-12.

27 Bressler NM, Boyer DS, Williams DF, et al. Cerebrovascular accidents in patients treated for choroidal neovascularization with ranibizumab in randomized controlled trials. Retina 2012:32:1821-8.

28 Avery RL, Francom SF, Lai P, et al. Meta-analysis examining the systemic safety profile of intravitreal ranibizumab injections in AMD, RVO, and DME. Invest Ophthalmol Vis Sci 2013:54:ARVO E-Abstract 1535.

29 European Medicines Agency. EPAR: Eylea (aflibercept) procedure no. EMEA/H/C/ 002392. http://www.ema.europa.eu/docs/en_GB/document_library/EPAR__Public assessment_report/human/002392/WC500135744.pdf (accessed 11 Mar 2014).

30 Avery RL, Pearlman J, Pieramici DJ, et al. Intravitreal bevacizumab (Avastin) in the treatment of proliferative diabetic retinopathy. Ophthalmology 2006;113:1695 e1-15.

31 Bakbak B, Ozturk BT, Gonul S, et al. Comparison of the effect of unilateral intravitreal bevacizumab and ranibizumab injection on diabetic macular edema of the fellow eye. I Ocular Pharmacol Ther 2013;29:728-32.

32 Avery RL. What is the evidence for systemic effects of intravitreal anti-VEGF agents, and should we be concerned? Br J Ophthalmol 2014;98(Suppl 1):i7-10. 